\title{
Pulmonary colonisation with Pneumocystis carinii in an immunosuppressed HIV-negative patient: detection and typing of the fungus by PCR
}

\author{
GILLES NEVEZ, KARINE GUYOT*, ANNE TOTET, CHRISTIAN RACCURT and EDUARDO DEI-CAS*
}

Department of Parasitology, Mycology and Travel Medicine, University of Picardy-University Hospital Centre, 80054 Amiens and *Department of Microbiology of Ecosystems, Pasteur Institute of Lille BP 245, 59019 Lille, and University Hospital Centre, Lille, France

\begin{abstract}
Mostly Pneumocystis carinii isolates from patients with acute pneumocystosis (PCP) have been typed until now. This report describes the typing of $P$. carinii organisms obtained from an HIV-negative patient without PCP. The patient underwent a broncho-alveolar lavage (BAL) to investigate an abnormal chest $\mathrm{X}$-ray. He was diagnosed with sarcoidosis. However, a low level of $P$. carinii organisms undetectable by microscopy was detected in BAL fluid by two subsequent nested PCR assays: one assay amplifying a portion of the mitochondrial large subunit RNA gene and a second one amplifying the internal transcribed spacers (ITS) 1 and ITS 2 of the nuclear rRNA operon. This low level of the fungus did not reflect acute PCP. Indeed, the clinical outcome was improvement despite the absence of specific treatment. The patient was considered to be only colonised by the fungus. Analysis of sequences of ITS PCR products led to identification of genotype Gg. This information constitutes the first data concerning $P$. carinii ITS genotype from a patient without acute PCP and HIV. This type has been described previously in AIDS patients diagnosed with PCP. These results show that PCR and ITS genotyping could represent efficient tools for the further investigation of the role played by HIV-negative patients with pulmonary colonisation in the human reservoir of $P$. carinii.
\end{abstract}

\section{Introduction}

Pneumocystis carinii remains the most frequent opportunist micro-organism causing severe pneumonia in immunosuppressed patients. Over the last decade, pulmonary carriage of $P$. carinii in healthy persons has been re-evaluated and $P$. carinii pneumonia (PCP) is now frequently considered to result from de novo infection rather than from reactivation of latent infection [1].

Nevertheless, low numbers of $P$. carinii organisms, undetectable by microscopy, have been detected by PCR assay on broncho-alveolar lavage (BAL) fluids from patients with no evidence of acute PCP. These low fungus levels were considered to reflect pulmonary colonisation. These cases of colonisation with $P$. carinii have been described mainly in patients with immunodeficiency and in those with pulmonary diseases [2-4].

Received 18 Feb. 2000; revised version received 18 June 2000; accepted 22 June 2000.

Corresponding author: Dr G. Nevez (e-mail: gnevez@ yahoo.fr).
As PCR is not usually used for the routine detection of $P$. carinii in pulmonary samples, diagnoses of cases of pulmonary colonisation with $P$. carinii are frequently missed. For this reason, and because the fungus is not easily cultivable, $P$. carinii strains responsible for colonisation have been typed in only one instance [5].

The aim of the present study was to describe the type of $P$. carinii obtained from an immunosuppressed HIVnegative patient with pulmonary colonisation. P. carinii was detected by PCR in a BAL fluid sample and was typed by PCR product sequencing. The genotype obtained from this patient without PCP was compared with genotypes described in recent reports concerning patients with acute PCP [6-8].

\section{Patient and methods}

Pulmonary colonisation was diagnosed in a 45-year-old patient who was submitted to BAL procedure to investigate pulmonary interstitial syndrome. The patient had no past history of PCP or HIV infection. Underlying conditions were diabetes and panhypopituitarism 
treated with hydrocortisone. The blood CD4+ T-cell count and the CD4 $+\mathrm{T}$ cell/CD8 $+\mathrm{T}$ cell ratio were $389 \times 10^{6} / \mathrm{L}$ and 0.46 , respectively.

P. carinii was not detected on BAL fluid sediment by methanol-Giemsa stain and an indirect immunofluorescence assay (IFA) with an anti- $P$. carinii cyst monoclonal antibody (Monofluo Kit ${ }^{\circledR}$ P. carinii, Diagnostics Pasteur, Marnes-La-Coquette, France). P. carinii DNA was detected by a hemi-nested PCR assay with a rapid DNA extraction procedure (GeneReleaser ${ }^{\circledR}$, BioVentures, Murfreesboro, TN, USA) and specific primers pAZ102-H and pAZ102-E (first PCR round), and pAZ102-L2 and pAZ102-E (second PCR round) amplifying a portion of the gene encoding the mitochondrial large subunit rRNA [9-10]. These PCR results were not used in patient management; however, the patient was followed-up for 12 months after the BAL procedure. As he did not develop PCP despite the absence of specific treatment for $P$. carinii, he was considered to be only colonised by $P$. carinii. Pulmonary sarcoidosis was finally diagnosed. Clinical improvement was obtained after corticosteroid treatment.

The fungus was typed by sequence analysis of the internal transcribed spacers (ITS1 and ITS2) of the nuclear rRNA operon. ITS types are defined by a combination of the alleles of the two loci ITS1 and ITS2. After a classical extraction procedure on the remaining BAL fluid sediment with proteinase $\mathrm{K}$ and phenol-chloroform, a second nested PCR was performed with the two pairs of primers NITSF and NITSR (first PCR round), and ITSF3 and ITS2R3 (second PCR round) [6,7]. The products of the second PCR round were cloned (pGEM-T vector System II, Promega Corporation, Madison, WI, USA) and sequenced from the two strands (Sequenase, Version 2.0, DNA sequencing kit, US Biochemical, Cleveland, $\mathrm{OH}$, USA). The sequences were compared with those reported by Tsolaki et al. [6,7] and Lee et al. [8].

\section{Results}

Considering sequence references and scores described for typing by Tsolaki et al. [6,7]; an ITS1 allele similar to allele A2 and A3 that was designated 'A', was found in the BAL fluid sample. However, it differed by $\mathrm{T}$ residues at positions 2 and 16. This allele ' $A$ ' was associated with ITS 2 allele a3. Thus, $P$ carinii genotype 'A'a3 was identified (Table 1). According to sequence references and applying the score of Lee et al. [8], genotype 'A'a3 was similar to genotype Gg.

\section{Discussion}

The possibility that the positive result of PCR detection could be related to sample contamination by fibroscopes previously used for patients with PCP was improbable. No case of acute PCP was noted during the same period. Moreover, as several negative controls were used (distilled water), contamination of samples by the airborne route in the laboratory, before and within PCR processing, was also excluded. Furthermore, $P$. carinii was detected by two nested PCR assays which were performed after two different extraction procedures and with specific primers from two different loci. These data were consistent with a genuine presence of the fungus in the respiratory tract of the patient. Nevertheless, for ethical reasons, no subsequent BAL was performed to confirm this presence.

Although this patient was not infected by HIV, he presented with an underlying pulmonary disease associated with an impairment of cellular immunity; the CD4+ T-cell count and the CD4 $+\mathrm{T}$ cell/CD8 $+\mathrm{T}$ cell ratio were $389 \times 10^{6} / \mathrm{L}$ and 0.46 , respectively $(\mathrm{N}$ $>600$ and $>1)$. It has been shown that an increasing risk of pulmonary colonisation with $P$. carinii in patients without HIV is associated with both a CD4+ $\mathrm{T}$ cell count $<400 \times 10^{6} / \mathrm{L}$ and a CD4 $+\mathrm{T}$ cell/CD8+ T-cell ratio $<1$ [4]. Thus, detection of $P$. carinii in this patient was consistent with his immune status.

Genotype Gg was identified in an immunosuppressed HIV-negative patient with colonisation. These are the first data concerning $P$. carinii ITS genotype from a patient without acute PCP. Genotype Gg was previously observed by Lee et al. [8] in Danish and American patients. All of these patients developed PCP and most of them were HIV-infected. This genotype was rarely observed in patients from the USA and Europe $5 \%$ of

Table 1. ITS1 and ITS2 sequences and genotype of $P$. carinii in an immunosuppressed HIV-negative patient without pneumocystosis

\begin{tabular}{l} 
ITS type* \\
\cline { 2 - 5 }
\end{tabular}


typed isolates), whereas the most frequent genotype was type $\mathrm{Eg}$ ( $20 \%$ of typed isolates) [8].

Genotype Gg was also found in one BAL fluid sample from a patient with PCP and AIDS, who was admitted to this hospital 5 weeks after the patient with sarcoidosis (data not shown). At present these results indicate that a similar $P$. carinii genotype could be found in an immunosuppressed HIV-negative patient with pulmonary colonisation and in patients with acute PCP and HIV. These results are consistent with those previously obtained using another typing method [5].

Host-to-host transmission of the micro-organism has been proved in rodents [11] and several observations suggested that inter-individual transmission occurred in man. Moreover, the host specificity of the fungus also pleads in favour of PCP as an anthroponosis. The above data are consistent with a human reservoir for $P$. carinii f. sp. hominis (human-derived P. carinii)

A recent study showed that the prevalence of pulmonary colonisation with $P$. carinii f. sp. hominis in patients with underlying pulmonary diseases and without HIV was $14 \%$ [4]. The hypothesis that this patient population could play a role as a reservoir of $P$. carinii f. sp. hominis cannot be ruled out and must be investigated. Although only one isolate was typed in the present study, results have shown that ITS genotyping of isolates from colonised patients could represent an efficient tool for further investigations concerning this hypothesis.

This work was partially supported by 'PRFMMIP' ('Programme de Recherche Fondamentale en Microbiologie et Maladies Infectieuses et Parasitaires') of the French Ministry of Education, Research and Technology.

\section{References}

1. Peters SE, Wakefield AE, Sinclair K, Millard PR, Hopkin JM. A search for Pneumocystis carinii in post-mortem lungs by DNA amplification. J Pathol 1992; 166: 195-198.

2. Leigh TR, Kangro HO, Gazzard BG, Jeffries DJ, Collins JV. DNA amplification by the polymerase chain reaction to detect sub-clinical Pneumocystis carinii colonization in HIV-positive and HIV-negative male homosexuals with and without respiratory symptoms. Respir Med 1993; 87: 525-529.

3. Tamburrini E, Mencarini P, De Luca A et al. Diagnosis of Pneumocystis carinii pneumonia: specificity and sensitivity of polymerase chain reaction in comparison with immunofluorescence in bronchoalveolar lavage specimens. J Med Microbiol 1993; 38: 449-453.

4. Nevez G, Raccurt C, Vincent P, Jounieaux V, Dei-Cas E. Pulmonary colonization with Pneumocystis carinii in human immunodeficiency virus-negative patients: assessing risk with blood $\mathrm{CD}^{+} \mathrm{T}$ cell counts. Clin Infect Dis 1999; 29: 13311332 .

5. Hauser PM, Blanc SD, Bille J, Nahimana A, Francioli P. Carriage of Pneumocystis carinii by immunosuppressed patients and molecular typing of the organisms. AIDS 2000; 14:461-463.

6. Tsolaki AG, Miller RF, Underwood AP, Banerji S, Wakefield AE. Genetic diversity at the internal transcribed spacer regions of the rRNA operon among isolates of Pneumocystis carinii from AIDS patients with recurrent pneumonia. J Infect Dis 1996; 174: 141-156.

7. Tsolaki AG, Beckers P, Wakefield AE. Pre-AIDS era isolates of Pneumocystis carinii f. sp. hominis: high genotypic similarity with contemporary isolates. J Clin Microbiol 1998; 36: $90-93$.

8. Lee C-H, Helweg-Larsen J, Tang $\mathrm{X}$ et al. Update on Pneumocystis carinii f. sp. hominis typing based on nucleotide sequence variations in internal transcribed spacer regions of rRNA genes. J Clin Microbiol 1998; 36: 734-741.

9. Wakefield AE, Pixley FJ, Banerji S et al. Detection of Pneumocystis carinii with DNA amplification. Lancet 1990; 336: $451-453$.

10. Rabodonirina M, Raffenot D, Cotte L et al. Rapid detection of Pneumocystis carinii in bronchoalveolar lavage specimens from human immunodeficiency virus-infected patients: use of a simple DNA extraction procedure and nested PCR. J Clin Microbiol 1997; 35: 2748-2751.

11. Soulez B, Palluault F, Cesbron J-Y, Dei-Cas E, Capron A, Camus D. Introduction of Pneumocystis carinii in a colony of SCID mice. J Protozool 1991; 38: 123S-125S. 